\title{
M olecular medicine, gene-expression profiling and molecular diagnostics: putting the cart before the horse
}

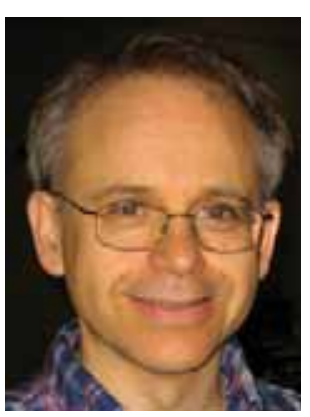

Staphen A Bustin, BA(Mod) PhD, Profesor of Mdealar Sience

Institute of Cell \& M olecular Science, Barts and the London School of M edicine and D entistry, Q ueen $M$ ary U niversity of London, UK; 3rd Floor, Alexandra Wing Royal London H ospital, London, E1 1BB, U K Tel.: +44 207377 7622; Fax: +44 207377 7283; E-mail: sa.bustin@ qmul.ac.uk

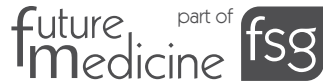

'...there have been scores of public a tions reporting an abundance of supposed individual marker genes or diverse gene lists that a re claimed to be associated with and even predic tive of patient survival.'

The terms 'molecular medicine' and '-omics technologies' encompass a range of procedures that are promising to help delineate more precisely pathophysiological mechanisms, identify individuals at risk for disease and suggest novel targets for drug treatment. The enhanced consistency and ease of use of core technologies such as real-time PCR ( leading to the incorporation of molecular analyses into clinical practice [1]; the introduction of next-generation sequencing technology [2], which permits massively parallel sequencing of millions of sequences at once, is likely to see an acceleration of this trend. This is particularly true in cancer research, where there is expectant acknowledgment that epigenetic, genetic and gene-expression profiling of primary tumors, cancers and/or circulating nucleic acids and tumor cells is a helpful and critical new weapon in the perpetual endeavor to advance and finetune our clinical diagnostic potential [3]. For example, there have been extensive attempts aimed at identifying genetic markers and metastasis-associated mRNA signatures in solid tumors, and using them as prognostic indicators of distant cancer recurrence [4]. Other common applications aim to identify mRNA signatures associated with cancer risk or track changes in mRN A levels linked with response to therapy [5]. Consequently, there have been scores of publications reporting an abundance of supposed individual marker genes or diverse gene lists that are claimed to be associated with and even predictive of patient survival [6-9]. Unfortunately, very few of these genes or gene lists agree with one another and it is striking that, several years after metastasis-associated gene lists were first published in a number of solid cancers, there is still no universal agreement on the utility of many of these markers for either determining prognosis or predicting response to therapy [10]. It is also essential to remember that an analysis of the genetic and expression profile of a tumor constitutes only one side of the equation. The other side is provided by the patient and it is clear that metastatic propensity is significantly influenced by host genetic background [11]. The identification of metastasis-predictive gene expression from normal tissue [12] means that it may be possible to identify individuals at increased risk of disseminated disease at the time of clinical manifestation of a primary cancer. Finally, molecular data are revealing the unappreciated intricacy of cellular regulatory pathways, for example, the complexity of genomic methylation patterns [13] that add to the difficulties in identifying clinically relevant molecular biomarkers.

Undeniably, the use of advanced technologies to define the molecular basis of malignancy has greatly improved our ability to stratify human cancers according to shared molecular characteristics and clinical behavior, with dramatic implications for clinical utility in cancer diagnosis, risk stratification and patient management [14]. This is particularly true for hematological cancers [15], with practical benefits less obvious for solid cancers $[16,17]$. H ere the clinical relevance of the extensive data generated remains unproven [18] and continues to await the results of largescale prospective validation studies that compare molecular against conventional markers [19].

In fairness, it is necessary to differentiate between technologies that target the genome, for example PCR, comparative genomic hybridization [20] and single nucleotide polymorphism microarrays [21], and those that target the transcriptome, for example RT-qPCR and mRN A/miRNA-expression microarrays. The utility, reliability and validity of the former is not in question, and enormous advances have been made in applying them to the discovery of new polymorphism, genetic and epigenetic alterations and novel classifications [22-24]. N evertheless, it is important to stress that factors that appear to predict metastatic disease are not necessarily the same ones that predict therapeutic 
response [25]. H owever, the real issue is with technologies that target RN A where, in my opinion, the translation of research results into clinical practice has been disappointing. There is an expectation that, ultimately, molecular biomarkers will prove to be of general use in clinical practice, and should improve both biologically guided therapeutic decisions and clinical outcomes. H ow likely is this? The understanding of clustered gene-expression patterns, the stratification of subgroups or the identification of entirely novel classes of common cancers, the testing of 'new' cancer genes for their prognostic or predictive value, or the study of any therapeutic value of new custom-designed drugs provides a formidable task, and cannot be accomplished without paying very close attention to the underlying technical, biological and clinical fundamentals. It may be necessary to manage our expectations: expression profiling of genes that causally contribute to elements of the metastatic process may identify useful drug targets for antimetastasis therapies [26], but may never be sufficiently accurate to replace conventional cancer staging procedures. Taking colorectal cancer as an example, I would like to discuss what I believe the main issues are and what needs to be done to correct the direction in which we are heading.

\section{Colorectal cancer}

Colorectal cancer is best described as a spectrum of diseases that is genetically heterogeneous and complicated [27]. Its heterogeneity is linked to the distinct genetic and epigenetic background of individual patients, coupled with the unique environment each host provides for tumor growth, mutation selection and subsequent metastasis. It is possible to stratify cancers by genetic status (e.g., genomic instability, microsatellite instability, genomic and microsatellite stability) as well as epigenetic status (e.g., CpG island methylator phenotype) that are relevant in determining clinical, biological and pathological characteristics of individual tumors [28]. The investigation of these molecular parameters has provided valuable clues to the cancers' pathogenesis, has allowed more accurate stratification of patients' risk of treatment failure and has resulted in the discovery of biomarkers that may be associated with more accurate assessment of treatment efficacy [29].

Geneexpression profiling, whilst extensive, has not been as successful. Several years of intensive research have identified a wide range of single genes and multiple gene lists that claim some association with the prediction of tumor behavior, but none are currently recommended for clinical use [30]. Unfortunately, results are not reproducible, few of the genes or gene lists are universally acknowledged as prognostic for individual patients and, taken together, there remains a wide gap between the delineation of molecular subtypes and their translation into diagnostic utility [3,31-43].

This, I believe, is due to a number of 'elephants' that, whilst known to be in the room, are conveniently ignored. O ne such elephant concerns the judicious application of our understanding of tumor biology and the kinetics of metastatic spread with the design of experimental protocols. This is coupled with what I perceive as the failure to assess whether the wealth of information obtained using molecular technologies is congruent with the actual world of practical medicine. A similar point has recently been made forcefully, describing biomedical research as an internally self-consistent universe with little contact with medical reality [44]. Another important factor in the handicapping of molecular diagnostics utilizing mRNAexpression profiling data is the lack of established quality control networks [45]. Quality assessment issues range from appropriate sample selection and handling, through RNA quality control, cDNA synthesis protocols, data handling and interpretation all the way to monitoring of the quality of the laboratories carrying out the molecular assays [46]. I consider there to be two sets of confounding parameters, broadly falling into biological and technical categories, which combine to make it difficult to characterize accurately or comprehensively a tumor from a single, small tissue biopsy.

\section{Confounding biological factors}

Tumor development requires a sequence of evolutionary steps, as deregulated growth and metastasis depends on successful adaptation to changing environmental constraints. Initial clonal expansion depends on selection mechanisms targeting gain-of-function mutations in oncogenes, loss-of-function mutations in tumor suppressor genes and disruption of normal senescence pathways. The initially dominant role of the microenvironment is subsequently complemented by competition for critical nutrients that results in selection pressure favoring phenotypes that increase substrate delivery or uptake. Individual mutations that increase the relative fitness of tumor cells have a high probability of 
becoming fixed in the main tumor. This could give rise to a primary tumor-associated metastasis profile. H owever, since the bulk of colorectal tumor is subdivided into clonal and/or spatial compartments, it is possible for metastasisassociated mutations to reach fixation in one compartment but not in the other.

- A colorectal cancer is composed of several individual clones that differ in their genotype and expression profile $[47,48]$. Tumor biopsies are unlikely to sample every one of these clones, and may not encompass the subclones that give rise to subsequent metastases. Consequently, it is uncertain how relevant it is to attempt to predict the behavior of a tumor made up of individual clones A-E from a genetic or expression profile unique to clone $B$, since it may be clone $E$ that has accumulated the genetic, epigenetic or expression characteristics facilitating the cancers ability to intravasate. It is interesting to note that the landmark publication describing the identification of a metastasis-associated gene signature in a number of solid tumors fails to include colorectal cancer [49].

- There is increasing evidence that the underlying cause of cancer is the deregulation of the normal stem cell regulatory system [50]. Stem cells constitute a very small percentage of a tumor's bulk [51]; during tumor progression, normal stem cells and their cellular pathways acquire stochastic malignant capacity, acquiring multiple genetic variants and resulting in genetically heterogeneous cancer stem cells [52]. This will generate subpopulations of cancer stem cells with a range of phenotypes that includes therapy-resistance, which allows the cancer to return after initial treatment as incurable metastatic disease. Clinical genomics data demonstrate that gene-expression signatures associated with the 'stemness' state of a cell can be informative as molecular predictors of cancer therapy outcome and can help to identify cancer patients with therapy-resistant tumors [53]. The number and location of stem cells is also important: patients with stem cells within the invasive margins of their tumor have a shorter average survival. This is also likely to make the identification of genuine metastasis-associated profiles more difficult as they are likely to be masked by the background noise emanating from the surrounding tumor mass. Indeed, there are reports of such signatures in normal tissue.
- mRNA levels continuously fluctuate, since mRN A transcription occurs in bursts and different $\mathrm{mRN}$ As are characterized by differential stability. An increasing number of genes are known to specify multiple splice variants that can be tissue specific and are translated into proteins with alternative and additional functions [54]. Most recently, it is emerging that genes can display allelic imbalance in their expression, so that allele-specific transcription results in the preferred expression of one or other of polymorphic variants [55]. This could be due to SN Ps in the gene sequence itself that could affect miRNA binding sites, or within the transcription factors transcribing it, or there could be differential DNA methylation around the genes' promoters. The simple targeting of part of an mRNA is no longer sufficient to be able to describe the expression of a particular gene. In addition, the mere presence of mRNA does not mean that it is being translated or, indeed, that a functional protein is being expressed. Finally, it has become clear that the presence and function of specific miRN As is as important to understanding gene expression as being able to quantitate the mRNA itself [56].

- It is also increasingly clear that the mere presence of tumor cells in tissue compartments such as blood [57] or lymph nodes [58] is not sufficient to allow an accurate prognosis for the individual patient. Leaving aside the question of tumor specificity of the detected mRNA, without knowledge of the metastatic potential such information merely identifies the tumor burden, which may or may not be related to the likelihood of metastasis development [46].

The analytical methods associated with molecular diagnostics require robust standard operating procedures coupled with extensive quality-assurance procedures. Unfortunately, many gene-expression data are seriously compromised by flagrant misuse of QPCR and microarray technologies, with the result that the biological uncertainties outlined previously are exacerbated by serious technical limitations.

\section{Confounding technical factors}

- Inappropriate sample selection, coupled with the complexity and heterogeneity of any tissue biopsy, especially from cancer and inconsistent handling procedures, results in 
variability and inaccurate $\mathrm{mRN} A$ quantification. In addition, there can be two sources of error: sampling error (i.e., even if epithelial cells are being collected, the cell type within the epithelial population may have a different distribution compared with the collected population) and measurement error, which depends on the quality of instruments, reagents and operator.

- The conversion of mRNA to CDNA is a major stumbling block and arguably is the single most variable step in the whole quantification procedure. It is well known, although not well publicized, that different reverse transcriptases have significantly different efficiencies of reverse transcription, and that these are target dependent $[59,60]$. Similarly, the mechanism of $C D N A$ priming has a significant effect on the outcome of any quantification experiment, since gene-specific priming, random priming and oligo-dT all produce diverse results that are distinct for different mR N A targets. The choice of primer location on the target mRNA can also yield significantly different results, as mRNA adopts a tight secondary structure characterized by extensive intrastrand base pairing, resulting in stem-loop structures [61]. If reverse transcription primers are designed to target stems, rather than loops, or if the amplicon can adopt secondary structures, the efficiency of the real-time step is significantly compromised. Characteristically, this results in unquantitative and unreproducible results.

- The accuracy of gene-expression profiling is highly dependent on mRNA quality $[62,63]$. Unfortunately, this is an area that is distinguished by a prevalent lack of concern. A 2005 survey of the working practices of 100 experienced $\mathrm{qPCR}$ users revealed that a worryingly high $37 \%$ did not quality assess their RN A, with a further $4 \%$ relying on absorbance ratios, which even then were known to be inadequate for quantification of $\mathrm{mRN} A$. In an attempt to consider current practices, I used the keyword 'real-time PCR' to identify 50 BM C publications in 2007/08 and looked at the ' $M$ ethods' section of every one. This revealed that we have regressed since 2005, with more than $60 \%$ of papers not even mentioning mRN A quality and a substantial $10 \%$ continuing to rely on absorbance ratio measurements. Even when RN A quality is assessed, it is evaluated using either gel electrophoresis or microfluidics-based systems; this approach fails to take into account that such measurements only look at ribosomal RNA without relating the results to $\mathrm{mRN} A$ integrity, which is, after all, the real target of interest. $N$ ot a single paper used the suggested $3^{\prime}: 5^{\prime}$ ratio of a panel of reference genes [63] or the target genes themselves.

- Splicing is a post-transcriptional modification in which a single gene can specify multiple proteins, allowing the synthesis of protein isoforms that are structurally and functionally distinct. Gene splicing affects most human genes [64] and plays an important role in human pathologies, including cancer [65]. This generates significant problems with the interpretation of RT-qPCR and microarray data, since presence or indeed significant changes in mRNA levels may reflect cell-, tissue or treatment-specific adjustments between different isoforms.

- The increased realization that allelic imbalance and allele-specific expression patterns are associated with increased disease risk [66,67] poses further problems for the interpretation of mRNA quantification data. Rather than avoiding SN Ps when designing primers, it may be necessary to include them as part of an overall assay design strategy so as to be able to quantitateallele-specific expression accurately.

- It is worth emphasizing that in vivo, mRN A is subject to constant degradation by complex interactions of deadenylation and decapping enzyme complexes as well as $3^{\prime}-5^{\prime}, 5^{\prime}-3^{\prime}$ exonucleases and endonucleases [68]. This is likely to result in significant natural variability of mRNA levels between genes expressed in different tissues and individuals. This is in addition to any degradation introduced during the extraction of the RNA from tissue samples or during storage. Whilst these comments may seem obvious, their implications have never been explored.

- Normalization, known to be an essential component of proper data analysis [69], continues to be used in an inappropriate manner, particularly in RT-qPCR applications, with a high proportion of papers still reporting expression patterns of target genes normalized against a single, invalidated reference gene.

- Inappropriate experimental designs, improper analyses, subjective interpretation of RT -qPCR data and variability of microarray results depending on the choice of analysis 
algorithms all combine to compromise the interpretation and confident application of quantitative, mR N A-targeted data [70].

\section{Conclusions}

The consequence of these, and other poor standards, is that a large number of publications report data that are at best unreliable and, at worst misleading, with a dramatic and damaging effect on the integrity of the scientific literature. For example, a paper published in Science and named as a 'breakthrough of the year' [71], has had to be withdrawn, because its results could not be repeated. More seriously, a paper using RT -qPC R technology and purporting to confirm an association between the presence of measles virus and gut pathology in children with developmental disorder [72] was used to claim a link between the MMR vaccine and autism [73]. $H$ owever, as last year's trial at the Vaccine Court in Washington DC, U SA, revealed, the data were significantly flawed as the RT-qPCR assay was applied in an inappropriate manner [101]. Importantly, two recent peer-reviewed publications have failed to reproduce any of the results underlying the claimed association, highlighting the challenges associated with translating this technology from a research context into a clinical diagnostic assay [74,75].

What is the solution? First, it is essential to step back and concentrate on getting the basic technical problems sorted out. This includes enforcing minimum quality standards for template preparation, validation and consistent use of CDNA priming methods, enzymes, protocols and, equally critically, appropriate analysis of data. Second, it is entirely unacceptable that most publications do not address the critical issue of RNA quality assessment. Third, it is equally unacceptable that data are not normalized in an appropriate manner. Fourth, it is crucial that data acquisition, analysis and reporting become more transparent. Consequently, it is necessary for the editors of scientific and biomedical publications to issue prescriptive checklists specifying the key information to be included when reporting experimental results. There are significant efforts underway to organize such 'minimum information' checklists, with the 'M inimum Information for Biological and Biomedical Investigations (MIBBI) project offering a common portal aimed at promoting gradual data integration [102].

Another development concerns the problems associated with attempting to share $\mathrm{QPCR}$ data between different laboratories and users. A new initiative, the 'Real-time PCR Data M arkup Language' (RDM L), describes a structured and universal data standard for exchanging $\mathrm{qPCR}$ data [103]. Together with the accompanying guidelines for M inimal Information qPCR, the data standard will contain sufficient information to understand the experimental set-up, reanalyze the data and interpret the results. This is of particular importance for transparent exchange of annotated GPCR data between authors, peer reviewers, journals and readers.

Finally, these approaches need to be combined with more suitable biological considerations, so that results are not a reflection of technical inadequacies and biological artifacts, but truly start reflecting actual differences in expression profiles between cells, tissues, individuals, disease states and treatment responses. Considerations of mRN A transcription, in vivo stability, regulation by miRN As, tissue specificity of splice variants, allele-specific differences in expression, the lack of concordance between most mRN As and their specified proteins, the critical importance of post-translational modifications, questions of tissue and tumor heterogeneity, the relative contributions of host and primary tumor to metastatic risk describe only a few serious issues that are not being addressed in an adequate manner in the rush to identify diagnostic, prognostic or therapeutic biomarkers.

Those intimately familiar with the molecular technologies underlying the advances proclaimed by the highest impact factor journals, and then taken up by the popular press and finally shaping peoples' expectations are only too familiar with their serious shortcomings. Remarkably, it seems that very few researchers are willing to listen and even fewer are willing to change their modi operandi. N evertheless, although it is not a popular cause, it is essential that we address these issues and it really is time to put the horse before the cart, and stop being blinded with ever-more technology.

Financial \& competing interests disclosure

The author was an expert witness for the U S D epartment of Justice at the Omnibus Autism Proceeding held at the U nited States C ourt of Federal Claimsin Washington DC in June 2007. The author has no other relevant affiliations or financial involvement with any organization or entity with a financial interest in or financial conflict with the subject matter or materials discussed in the manuscript apart from those disclosed.

$\mathrm{No}$ writing assistance was utilized in the production of this manuscript. 
Bibliography

1. Roukos DH, M urray S, Briasoulis E: M olecular genetic tools shape a roadmap towards a more accurate prognostic prediction and personalized management of cancer. Cancer Biol. Ther. 6(3), 308-312 (2007).

2. Church $\mathrm{GM}$ : G enomes for all. Sci. Am. 294(1), 46-54 (2006).

3. Yamasaki M, Takemasa I, Komori T et al.: The gene expression profile represents the molecular nature of liver metastasis in colorectal cancer. Int. J. O ncol. 30(1), 129-138 (2007).

4. Wang Y, Klijn J, Zhang Y, Atkins D, Foekens $\mathrm{J}$ : Gene expression profiles and prognostic markers for primary breast cancer. M ethods M ol. Biol. 377, 131-138 (2007).

5. Pramana J, Van den Brekel M W, van Velthuysen $M L$ et al.: G ene expression profiling to predict outcome after chemoradiation in head and neck cancer. Int. J. Radiat. O ncol. Biol. Phys. 69(5), 1544-1552 (2007).

6. Mazumder $A$, Wang $Y$ : G ene-expression signatures in oncology diagnostics. Pharmacogenomics 7(8), 1167-1173 (2006).

7. Thomassen $M$, Tan $Q$, Eiriksdottir F, Bak $M, C$ old S, Kruse TA: Prediction of metastasis from low-malignant breast cancer by gene expression profiling. Int. J. Cancer 120(5), 1070-1075 (2007).

8. Foukakis T, Gusnanto A, Au AY et al.: A PCR-based expression signature of malignancy in follicular thyroid tumors. Endocr. Relat. Cancer 14(2), 381-391 (2007).

9. Wiese $A H$, Auer J, Lassmann $S$ et al.: Identification of gene signatures for invasive colorectal tumor cells. Cancer D etect. Prev. 31(4), 282-295 (2007).

10. D uffy $M J$, van $D$ alen $A, H$ aglund $C$ et al.: Tumour markers in colorectal cancer: European Group on Tumour M arkers (EGTM ) guidelines for clinical use. Eur. J. Cancer 43(9), 1348-1360 (2007).

11. H unter $\mathrm{K}$ : $\mathrm{H}$ ost genetics influence tumour metastasis. Nat. Rev. Cancer 6(2), 141-146 (2006).

12. Yang $H$, Crawford $N$, Lukes L, Finney $R$, Lancaster $M, H$ unter $K W$ : M etastasis predictive signature profiles preexist in normal tissues. Clin. Exp. M etastasis 22(7), 593-603 (2005).

13. Korshunova $\mathrm{Y}, \mathrm{M}$ aloney RK, Lakey $\mathrm{N}$ et al.: $M$ assively parallel bisulphite pyrosequencing reveals the molecular complexity of breast cancer-associated cytosine-methylation patterns obtained from tissue and serum DN A. Genome Res 18(1), 19-29 (2008).
14. He M, Rosen J, M angiameli D, Libutti SK: Cancer development and progression. Adv. Exp. M ed. Biol. 593, 117-133 (2007).

15. Glinsky GV, Berezovska O, G linskii AB: $M$ icroarray analysis identifies a death-fromcancer signature predicting therapy failure in patients with multiple types of cancer. J. Clin. Invest. 115(6), 1503-1521 (2005).

16. Sotiriou C, Piccart M J: Taking geneexpression profiling to the clinic: when will molecular signatures become relevant to patient care? $\mathrm{N}$ at. Rev. Cancer 7(7), 545-553 (2007).

17. Koscielny S: Critical review of microarraybased prognostic tests and trials in breast cancer. Curr. O pin. O bstet. Gynecol. 20(1), 47-50 (2008).

18. Lusa L, M CShane LM, Reid JF et al.: Challenges in projecting clustering results across gene expression-profiling datasets. J. N atl Cancer Int. 99(22), 1715-1723 (2007).

19. Bustin SA, D orudi S: The value of microarray techniques for quantitative gene profiling in molecular diagnostics. Trends M ol. M ed. 8(6), 269-272 (2002).

20. Aradhya S, Cherry AM : Array-based comparative genomic hybridization: clinical contexts for targeted and wholegenome designs. Genet. M ed. 9(9), 553-559 (2007).

21. Wang L, Luhm R, Lei M : SN P and mutation analysis. Adv. Exp. M ed. Biol. 593, 105-116 (2007).

22. Kopper L, Timar J: G enomics of lung cancer may change diagnosis, prognosis and therapy. Pathol. O ncol. Res. 11(1), 5-10 (2005).

23. Barton $C A, H$ acker $N F, C$ lark SJ, O 'Brien PM : D N A methylation changes in ovarian cancer: implications for early diagnosis, prognosis and treatment. Gynecol. 0 ncol. 109(1), 129-139 (2008).

24. Araujo SE, Bernardo W M , H abr-G ama A, Kiss DR, Cecconello I: D N A ploidy status and prognosis in colorectal cancer: a metaanalysis of published data. $D$ is. Colon Rectum 50(11), 1800-1810 (2007).

25. Jeffrey SS, Lonning PE, $\mathrm{H}$ illner $B E$ : Genomics-based prognosis and therapeutic prediction in breast cancer. J. $\mathrm{N}$ atl $\mathrm{Compr}$. Canc. N etw. 3(3), 291-300 (2005).

26. Fingleton B: M olecular targets in metastasis: lessons from genomic approaches. Cancer Genomics Proteomics 4(3), 211-221 (2007).

27. Takayama T, M iyanishi $K, H$ ayashi $T$, Sato Y, N iitsu Y: Colorectal cancer: genetics of development and metastasis. J. Gastroenterol. 41(3), 185-192 (2006).
28. Chang SC, Lin JK, Yang SH, Wang H S, Li $\mathrm{AF}, \mathrm{Chi} \mathrm{CW}$ : Relationship between genetic alterations and prognosis in sporadic colorectal cancer. Int. J. Cancer 118(7), 1721-1727 (2006).

29. O gino $S, G$ oel $A$ : M olecular classification and correlates in colorectal cancer. J. M Ol. Diagn. 10(1), 13-27 (2008).

30. Locker $\mathrm{GY}, \mathrm{H}$ amilton $\mathrm{S}, \mathrm{H}$ arris J et al.: ASCO 2006 update of recommendations for the use of tumor markers in gastrointestinal cancer. J. Clin. 0 ncol. 24(33), 5313-5327 (2006).

31. Al-M ulla F, Behbehani AI, Bitar M S, Varadharaj G, Going JJ: G enetic profiling of stage I and II colorectal cancer may predict metastatic relapse. M od. Pathol. 19(5), 648-658 (2006).

32. Shih W, Chetty R, Tsao M S: Expression profiling by microarrays in colorectal cancer. Oncol. Rep. 13(3), 517-524 (2005).

33. Eschrich S, Yang I, Bloom $\mathrm{G}$ et al.: M olecular staging for survival prediction of colorectal cancer patients. J. Clin. O ncol. 23(15), 3526-3535 (2005).

34. Chiu ST, H sieh FJ, Chen SW, Chen CL, Shu H F, Li H : Clinicopathologic correlation of up-regulated genes identified using CD N A microarray and real-time reverse transcription-PCR in human colorectal cancer. Cancer Epidemiol. Biomarkers Prev. 14(2), 437-443 (2005).

35. Arango D, Laiho P, Kokko $A$ et al.: Geneexpression profiling predicts recurrence in D ukes' C colorectal cancer. Gastroenterology 129(3), 874-884 (2005).

36. Kwon H C, Kim SH, Roh M S et al.: Gene expression profiling in lymph node-positive and lymph node-negative colorectal cancer. Dis. Colon Rectum 47(2), 141-152 (2004).

37. Williams N S, Gaynor RB, Scoggin S et al.: Identification and validation of genes involved in the pathogenesis of colorectal cancer using CD N A microarrays and RN A interference. Clin. Cancer Res. 9(3), 931-946 (2003).

38. Frederiksen CM, Knudsen S, Laurberg $S$, Orntoft TF: Classification of Dukes' $B$ and $C$ colorectal cancers using expression arrays. J. Cancer Res. Clin. Oncol. 129(5), 263-271 (2003).

39. Stremmel $C$, Wein $A, H$ ohenberger $W$, Reingruber B: DN A microarrays: a new diagnostic tool and its implications in colorectal cancer. Int. J. Colorectal Dis. 17(3), 131-136 (2002).

40. Birkenkamp-D emtroder K, Christensen LL, O lesen $\mathrm{SH}$ et al.: G ene expression in colorectal cancer. Cancer Res.62(15), 4352-4363 (2002). 


\section{M olecular medicine, gene-expression profiling \& molecular diagnostics - EDIT O RIAL}

41. Yanagawa R, Furukawa Y, Tsunoda T et al.: $G$ enome-wide screening of genes showing altered expression in liver metastases of human colorectal cancers by CD N A microarray. N eoplasia 3(5), 395-401 (2001).

42. Takemasa I, H iguchi $\mathrm{H}$, Yamamoto $\mathrm{H}$ et al.: Construction of preferential $C D N A$ microarray specialized for human colorectal carcinoma: molecular sketch of colorectal cancer. Biochem. Biophys. Res Commun. 285(5), 1244-1249 (2001).

43. H egde P, Q i R, G aspard R et al.: Identification of tumor markers in models of human colorectal cancer using a 19,200-element complementary D N A microarray. Cancer Res. 61(21), 7792-7797 (2001).

44. H orrobin D F: M odern biomedical research: an internally self-consistent universe with little contact with medical reality? $\mathrm{N}$ at. Rev. 2(2), 151-154 (2003).

45. van der Velden VH, Panzer-Grumayer ER, Cazzaniga $G$ et al.: 0 ptimization of PCRbased minimal residual disease diagnostics for childhood acute lymphoblastic leukemia in a multi-center setting. Leukemia 21(4), 706-713 (2007).

46. Bustin SA, M ueller R: Real-time reverse transcription PCR and the detection of occult disease in colorectal cancer. M ol. Aspects M ed. 27, 192-223 (2006).

47. Baisse $B$, Bouzourene $H$, Saraga EP, Bosman FT, Benhattar J: Intratumor genetic heterogeneity in advanced human colorectal adenocarcinoma. Int.j. Cancer 93(3), 346-352 (2001).

48. Losi L, Baisse B, BouzoureneH, Benhattar J: Evolution of intratumoral genetic heterogeneity during colorectal cancer progression. Carcinogenesis 26(5), 916-922 (2005).

49. Ramaswamy S, Ross KN, Lander ES, G olub TR: A molecular signature of metastasis in primary solid tumors. $\mathrm{N}$ at. Genet. 33(1), 49-54 (2003).

50. Brabletz $T$, Jung A, Spaderna S, H lubek F, Kirchner $\mathrm{T}$ : O pinion: migrating cancer stem cells: an integrated concept of malignant tumour progression. N at. Rev. Cancer 5(9), 744-749 (2005)

51. Ricci-Vitiani L, Lombardi DG, Pilozzi E et al.: Identification and expansion of human colon-cancer-initiating cells. N ature 445(7123), 111-115 (2007).

52. Lagasse $\mathrm{E}$ : $\mathrm{C}$ ancer stem cells with genetic instability: the best vehicle with the best engine for cancer. GeneT her. 15(2), 136-142 (2008).
53. G linsky GV: Stem cell origin of death-fromcancer phenotypes of human prostate and breast cancers. Stem Cell Rev. 3(1), 79-93 (2007).

54. de la Grange P, D utertre M, Correa M , Auboeuf $D: A$ new advance in alternative splicing databases: from catalogue to detailed analysis of regulation of expression and function of human alternative splicing variants. BM C Bioinformatics 8, 180 (2007).

55. Bjornsson H T, Albert TJ, Ladd-Acosta CM et al.: SN P-specific array-based allele-specific expression analysis. Genome Res. 18(5), 771-779 (2008).

56. Landi D, Gemignani F, N accarati $A$ et al.: Polymorphisms within micro-R N A-binding sites and risk of sporadic colorectal cancer. Carcinogenesis 29(3), 579-584 (2008).

57. Schuster R, M ax N, M ann B et al.: $Q$ uantitative real-time RT-PCR for detection of disseminated tumor cells in peripheral blood of patients with colorectal cancer using different mRN A markers. Int. J. Cancer 108(2), 219-227 (2004).

58. Bustin SA, D orudi S: G ene expression profiling for molecular staging and prognosis prediction in colorectal cancer. Expert Rev. M ol. Diagn. 4(5), 599-607 (2004).

59. Stahlberg A, H akansson J, Xian X, Semb H, Kubista M : Properties of the reverse transcription reaction in $\mathrm{mRN} A$ qualification. Clin. Chem. 50(3), 509-515 (2004).

60. Stahlberg A, Kubista M, Pfaffl M : Comparison of reverse transcriptases in gene expression analysis. Clin. Chem. 50(9), 1678-1680 (2004).

61. Bustin SA, N olan T: Pitfalls of quantitative real-time reverse-transcription polymerase chain reaction. J. Biomol. Tech. 15(3), 155-166 (2004).

62. N olan T, H ands RE, O gunkolade BW, Bustin SA: SPUD : a qPCR assay for the detection of inhibitors in nucleic acid preparations. Anal. Biochem. 351, 308-310 (2006).

63. N olan $\mathrm{T}, \mathrm{H}$ ands RE, Bustin SA: Q uantification of mRN A using real-time RTPCR. N at. Protoc. 1(3), 1559-1582 (2006).

64. Ben-D ov C, H artmann B, Lundgren J, Valcarcel J: G enome-wide analysis of alternative pre-mRN A splicing. J. Biol. Chem. 283(3), 1229-1233 (2008).

65. Pettigrew CA, Brown M A: PremRN A splicing aberrations and cancer. Front. Biosci. 13, 1090-1105 (2008)

66. Meyer KB, M aia AT, $O$ 'Reilly $M$ et al.: Allele-specific up-regulation of FGFR2 increases susceptibility to breast cancer. PL oS Biol. 6(5), el08 (2008).
67. Chen X, Weaver J, Bove BA et al.: Allelic imbalance in BRCA1 and BRCA2 gene expression is associated with an increased breast cancer risk. H um. M ol. Genet. 17(9), 1336-1348 (2008).

68. Coller J, Parker R: Eukaryotic mRN A decapping. Annu. Rev. Biochem. 73, 861-890 (2004).

69. Vandesompele J, D e Preter K, Pattyn F et al.: Accurate normalization of real-time quantitative RT-PCR data by geometric averaging of multiple internal control genes. Genome Biol. 3(7), 0034.0031-0034.0011 (2002).

70. Bustin SA, M ueller R: Real-time reverse transcription PCR ( $q R T-P C R$ ) and its potential use in clinical diagnosis. Clin. Sci. 109(4), 365-379 (2005).

71. H uang T, Bohlenius H, Eriksson S, Parcy F, $\mathrm{N}$ ilsson 0 : The mRN A of the Arabidopsis gene FT moves from leaf to shoot apex and induces flowering. Science 309(5741), 1694-1696 (2005).

72. U hlmann $\mathrm{V}, \mathrm{M}$ artin $\mathrm{CM}$, Sheils $\mathrm{O}$ et al.: Potential viral pathogenic mechanism for new variant inflammatory bowel disease. $\mathrm{M} \mathrm{ol}$. Pathol. 55(2), 84-90 (2002).

73. Bradstreet J J, El D ahr J, Anthony A Kartzinel JJ, Wakefield AJ: D etection of measles virus genomic RN A in cerebrospinal fluid of children with regressive autism: a report of three cases. J. Am. Phys. Surg. 9, 38-45 (2004).

74. Afzal M A, O zoemena $L C, O{ }^{\prime} H$ are $A$, Kidger KA, Bentley M L, M inor PD: Absence of detectable measles virus genome sequence in blood of autistic children who have had their M M R vaccination during the routine childhood immunization schedule of UK. J. M ed. Virol. 78(5), 623-630 (2006).

75. D'Souza Y, D ionne S, Seidman EG, Bitton A, Ward BJ: N o evidence of persisting measles virus in the intestinal tissues of patients with inflammatory bowel disease. Gut 56(6), 886-888 (2007).

Websites

101. United States C ourt of Federal Claims: Vaccine Program/O ffice of Special M asters. ftp://autism.uscfc.uscourts.gov/autism/ cedillo.html

102. MIBBI: M inimum Information for Biological and Biomedical Investigations. http://mibbi.sourceforge.net

103. RD M L: Real-time PCR D ata M arkup Language. www.rdml.org/ 